\section{A Randomized, Double-Blinded, Placebo- Controlled, Cross Over Study Evaluating the Efficacy and Safety of Timolol Ophthalmic Solution as an Acute Treatment of Migraine}

Dipika Aggarwal, M.D., Andrew J. Heim, CCRP,

Brennen Bittel, D.O., Deetra Ford, M.D.,

Richard Dubinsky, M.D., Gary Gronseth, M.D.,

Vernita Hairston, M.D., Patrick Landazuri, M.D.,

Fred Sachen, M.D., Laura Herbelin, CCRP,

Richard J. Barohn, M.D.

University of Kansas Medical Center, Kansas City, KS

Department of Neurology

Received Feb. 10, 2020; Accepted for publication Feb. 10, 2020; Published online Feb. 26, 2020

\begin{abstract}
Introduction. Daily oral beta-adrenoreceptor antagonist has been shown to be effective in preventing migraine headaches. Timolol $0.5 \%$ ophthalmic solution is a non-selective beta-adrenoreceptor antagonist, where the primary use is for glaucoma. There have been case reports that timolol is effective in aborting or improving an acute migraine headache. The objective of this study was to assess the efficacy (decrease of $\geq 50 \%$ in pain scale at 120 minutes) of timolol $0.5 \%$ ophthalmic solution compared to placebo in acute treatment of migraine headache.
\end{abstract}

Methods. We performed a randomized, double-blind, crossover, placebo-controlled, study. Study entry criteria required subjects to have one to eight migraine episodes per month. The primary outcome was comparison of the change in a visual analog pain scale (VAS) at 120 minutes after taking the study medication. Study subjects were given a pain scale with a range of 1 (no pain) to 10 (most severe pain) to complete after onset of migraine but before administration of study drops and 120 minutes after administration of study drops. Improvement was defined as a $\geq 50 \%$ decrease in pain scale.

Results. Nineteen subjects completed the study and were used for analysis. The primary outcome changes in pain scale, 120 minutes after dose, showed a similar decrease for placebo and drug with a slightly wider $95 \%$ CI for placebo. Six subjects in each arm experienced $\mathrm{a} \geq 50 \%$ decrease in pain scale.

Conclusion. These results support that timolol $0.5 \%$ ophthalmic solution is not an efficacious treatment for acute migraine headache.

Kans J Med 2020;13(Suppl 2):2-5.

\section{INTRODUCTION}

Migraine is a common, disabling neurological disorder, affecting 10 $12 \%$ of the U.S. population; $18 \%$ women and $6 \%$ men. ${ }^{1}$ The treatment of migraine headache includes preventive therapies and abortive therapies. There are several preventive therapies available with the goal to decrease the overall frequency and intensity of migraine attacks. The abortive medications are used during acute migraine episode. There are several methods that are used for migraine abortive therapy, such as triptans and NSAIDs.

Despite the various migraine abortive therapies available, there is an ongoing need for novel therapies. Beta-blocker medications have

\section{KANSAS JOURNAL of MEDICINE}

been used for migraine prevention for decades. However, their use for migraine abortion has not been well-identified.

Oral beta-blockers are a class of medications frequently used to control blood pressure, angina, and heart irregularities. Certain oral beta-blockers, such as propranolol, are used on a daily basis to prevent migraines. Both topical and oral timolol can be used daily prophylactically to decrease the frequency of migraine..$^{2-5}$ However, propranolol and timolol tablets have not been shown to be effective as an acute treatment to stop attacks of migraine because of their longer onset of action.

In this study, we tested the use of topical beta-blocker eye drops for acute treatment of migraine headache. It was proposed that betablocker eye drops, since they are quickly absorbed unlike tablets, can be beneficial and efficacious in the treatment of headache abortion. A prior case series of seven cases reported a substantial benefit of beta blocker eye drops in acute migraine in all patients. ${ }^{6}$ The case series was published with four editorials in the same journal recommending that further studies be done in the form of randomized-controlled trials. ${ }^{7-10}$ We now report a randomized, placebo-controlled, blindedcontrolled trial to test the use of timolol for acute migraine headache.

\section{METHODS}

Trial Design. We performed a randomized, double-blind, crossover, placebo-controlled, study containing a screening visit, a double-blind treatment phase, and an end-of-study visit. The University of Kansas Medical Center was the only site as part of the study that enrolled patients from April 2017 to February 2018.

Standard Protocol Approvals, Registrations, and Patient Consents. The trial was approved by the Institutional Review Board at the University of Kansas Medical Center. Written informed consent was obtained by all participants, in accordance with the Declaration of Helsinki and the principles of Good Clinical Practice. This study was registered at clinicaltrials.gov (NCT03836664).

A total of 26 subjects were enrolled in a randomized, double-blind fashion. Participants ranged in age from 22 - 64 years; 25 female and one male. Subjects enrolled had a diagnosis of migraine with or without aura according to ICHD-2 criteria for at least one year prior to informed consent. Frequency of migraine episode was between one to eight migraines per month for inclusion. Women of childbearing potential had to complete a negative pregnancy test and practice effective birth control methods throughout the duration of the study.

Patients were excluded if they were not able to distinguish between migraines and headaches; had headaches at a frequency greater than 15 days per month; used chronic opioid therapy for headaches; had a history or signs and symptoms of ischemic cardiac syndromes; a history of glaucoma and/or current treatment with prescription eye drops; history of naso-lacrimal duct obstruction or surgery; treatment for any severe ophthalmic disease; physical problems or coordination difficulty or eye avoidance sensitivity that would preclude proper 
KANSAS JOURNAL of MEDICINE

OPTHAMOLIC TIMOLOL FOR MIGRAINE

continued.

installation of eye drops; history of uncontrolled asthma, COPD, diabetes mellitus, hyperthyroidism, or reversible airway disease; history of clinically symptomatic bradycardia, congestive heart failure, or hypotension; and a history of drug or alcohol abuse as defined by DSM-IV criteria.

Baseline characteristics include the location, symptom, and character of migraines experienced by enrolled subjects. Frontal was the most common location migraines were experienced at $42 \%$ of study population. The different characters associated with migraines experienced by the study population were throbbing, pulsating, sharp, stabbing, and pressure. Symptoms associated with migraines were also recorded. Symptoms recorded were photophobia, nausea, phonophobia, and visual.

Outcomes and Measures. Baseline characteristics included gender, age, and ethnicity. Migraine history was also collected including frequency, location, character, and associated symptoms. Subject-reported pain scales, satisfaction questionnaires, adverse events, and concomitant medications were assessed at visit two and visit three.

The primary end point of the study is to compare differences in headache severity at 120 minutes between study medication and placebo. Study subjects were given a visual analog pain scale (VAS) with a range of 0 (no pain) to 10 (most severe pain) to complete at two different time points: at onset of migraine and before administration of study drops, and at 120 minutes after administration of study drops. To account for the crossover design, the mean difference in the change in headache severity following placebo compared to timolol for each patient along with its $95 \%$ confidence interval was calculated.

The secondary end points were to assess subject satisfaction with treatment, proportion of subjects with at least a $50 \%$ decreased in pain severity at two hours, and safety. Patients were given a Treatment Satisfaction Scale to be completed 120 minutes after administration of study drops. The Treatment Satisfaction Scale contained five items: not satisfied at all, somewhat not satisfied, neutral, somewhat satisfied, and very satisfied. Adverse events were recorded at two timepoints throughout the study for safety.

Randomization and Blinding. Patients were randomly assigned to timolol $0.5 \%$ solution or placebo based on their subject number at the baseline visit. Subjects were assigned study IDs in the numerical order they were enrolled. Consistent with the design of the crossover study, patients that did not receive timolol $0.5 \%$ solution at the baseline visit were given timolol at visit two and vice versa. The investigator, study staff, and subjects were blinded to treatment assignment.

Sample Size and Calculation. A total of 26 subjects were enrolled in the study. Twenty subjects were needed to have an $80 \%$ chance of detecting a one-point difference in the change in pain scores between the two arms, at an alpha of 0.05 .

\section{RESULTS}

Twenty-eight patients were screened for eligibility; 26 were enrolled. Reasons for screen failure included headache frequency greater than 15 episodes per month and not experiencing a migraine within a month after being enrolled. One patient dropped out of the study and six patients did not complete the study. Nineteen total participants completed the study and were used for analysis.

Out of the 26 subjects enrolled, participants were nearly exclusively female (96\%) and an average age of 41 years (range 21 - 63). Participants averaged three migraine episodes per month with a range of one to eight episodes per month. The most common character of migraines was throbbing (96\%) and nausea (77\%) was the most common symptom (Table 1 ).

Table 1. Baseline characteristics of migraines.

\begin{tabular}{|l|c|c|}
\hline Symptom & Number & \% \\
\hline Photophobia & 25 & 96 \\
\hline Nausea & 20 & 77 \\
\hline Phonophobia & 13 & 50 \\
\hline Visual & 2 & 8 \\
\hline Location & Number & $\%$ \\
\hline Frontal & 11 & 42 \\
\hline Occipital & 8 & 31 \\
\hline Holocephalic & 8 & 31 \\
\hline Temporal & 4 & 15 \\
\hline Character & Number & $\%$ \\
\hline Throbbing & 25 & 96 \\
\hline Pulsating & 6 & 23 \\
\hline Sharp & 4 & 15 \\
\hline Stabbing & 3 & 12 \\
\hline Pressure & 1 & 4 \\
\hline
\end{tabular}

Table 2 shows the raw data. Pain reductions were not significantly different following placebo or timolol treatment. Table 3 shows the mean difference of differences in change in pain, 0.1 (SD 2.6) VAS points favoring placebo, 95\% CI -1.1 to 1.3. Similarly, there were no significant differences in the number of patients reporting $50 \%$ or more reduction in pain severity (six for each treatment) or for satisfaction with treatment (timolol $=3$, placebo $=6$ ).

One adverse event was experienced throughout the study, which was a mild stinging/burning sensation in the eye shortly after administration of eye drops. Each was graded mild, related, and all subjects recovered. Four of 19 study completers experienced this adverse event. 
Table 2. Summary of subject responses while using timolol and placebo.

\begin{tabular}{|c|c|c|c|c|c|c|}
\hline \multicolumn{7}{|l|}{ Drug } \\
\hline Subject number & Pain scale before drug & Pain scale 120 min. after drug & Delta & Percent decrease & $\geq 50 \%^{\mathrm{a}}$ & Satisfied $^{a}$ \\
\hline MOO1 & 7 & 3 & 4 & 57 & 1 & 0 \\
\hline M003 & 3 & 0 & 3 & 100 & 1 & 1 \\
\hline M004 & 9 & 8 & 1 & 11 & 0 & 0 \\
\hline M006 & 8 & 6 & 2 & 25 & 0 & 0 \\
\hline M007 & 8 & 8 & 0 & 0 & 0 & 0 \\
\hline MO09 & 8 & 8 & 0 & 0 & 0 & 0 \\
\hline M010 & 4 & 6 & -2 & -50 & 0 & 0 \\
\hline M011 & 6 & 6 & 0 & 0 & 0 & 0 \\
\hline MO12 & 8 & 3 & 5 & 63 & 1 & 0 \\
\hline M013 & 9 & 4 & 5 & 56 & 1 & 0 \\
\hline M014 & 10 & 4 & 6 & 60 & 1 & 1 \\
\hline M016 & 9 & 7 & 2 & 22 & 0 & 0 \\
\hline M017 & 7 & 7 & 0 & 0 & 0 & 0 \\
\hline M019 & 7 & 6 & 1 & 14 & 0 & 0 \\
\hline M021 & 8 & 5 & 3 & 38 & 0 & 0 \\
\hline MO22 & 5 & 4 & 1 & 20 & 0 & 0 \\
\hline M023 & 8 & 4 & 4 & 50 & 1 & 1 \\
\hline MO24 & 7 & 5 & 2 & 29 & 0 & 0 \\
\hline M025 & 7 & 6 & 1 & 14 & 0 & 0 \\
\hline \multicolumn{7}{|l|}{ Placebo } \\
\hline MOO1 & 7 & 3 & 4 & 57 & 1 & 1 \\
\hline M003 & 2 & 2 & 0 & 0 & 0 & 0 \\
\hline M004 & 7 & 4 & 3 & 43 & 0 & 0 \\
\hline M006 & 9 & 3 & 6 & 67 & 1 & 1 \\
\hline M007 & 7 & 4 & 3 & 43 & 0 & 1 \\
\hline M009 & 8 & 8 & 0 & 0 & 0 & 0 \\
\hline M010 & 5 & 7 & -2 & -40 & 0 & 0 \\
\hline M011 & 5 & 4 & 1 & 20 & 0 & 0 \\
\hline M012 & 7 & 1 & 6 & 86 & 1 & 1 \\
\hline M013 & 9 & 5 & 4 & 44 & 0 & 0 \\
\hline M014 & 8 & 9 & -1 & -13 & 0 & 0 \\
\hline M016 & 9 & 4 & 5 & 56 & 1 & 1 \\
\hline M017 & 6 & 7 & -1 & -17 & 0 & 0 \\
\hline M019 & 6 & 7 & -1 & -17 & 0 & 0 \\
\hline M021 & 7 & 5 & 2 & 29 & 0 & 0 \\
\hline M022 & 6 & 6 & 0 & 0 & 0 & 0 \\
\hline M023 & 4 & 2 & 2 & 50 & 1 & 0 \\
\hline M024 & 7 & 2 & 5 & 71 & 1 & 1 \\
\hline M025 & 7 & 7 & 0 & 0 & 0 & 0 \\
\hline
\end{tabular}

${ }^{\mathrm{a}} \geq 50 \%$ decrease and satisfaction $\operatorname{coded} 0=$ no, $1=$ yes.

Table 3. Change in VAS.*

\begin{tabular}{|c|c|c|c|c|c|}
\hline \multicolumn{2}{|l|}{ Timolol } & \multicolumn{3}{l|}{ Placebo } \\
\hline Onset & Two hours & Delta & Onset & Two hours & Delta \\
\hline $7.3(1.7)$ & $5.3(2.0)$ & $2(2.1)$ & $6.63(1.7)$ & $4.7(2.2)$ & $1.9(2.5)$ \\
\hline
\end{tabular}


KANSAS JOURNAL of MEDICINE OPTHAMOLIC TIMOLOL FOR MIĞRANE continued.

\section{DISCUSSION}

Migraine and other benign recurrent headache disorders are a major public health problem, particularly among reproductive-aged women. They are associated with substantial personal suffering, disability, and societal expense. The impact of migraine is substantial because of its high prevalence, accompanying significant disability, and risk for other comorbidities. Data from the National Ambulatory Medical Care Survey (NAMCS), and the National Hospital Ambulatory Medical Care Survey (NHAMCS) indicate that headache is among the top 20 reasons for outpatient medical visits and among the top five reasons for emergency department visits. Several preventive and abortive medications are available for the treatment of migraine headaches. Despite of various treatments available, there seem to be an ongoing need for novel therapies.

Certain beta-blockers medications like propranolol and timolol have been used as a preventive therapy for migraine headaches. Their safety and efficacy in the prevention of migraines has been well established. In this study we used topical beta-blocker eye drops for acute treatment of migraine attack. Topical and local application betablocker eye drops are directly absorbed through the ophthalmic and nasolacrimal mucosa and enters the blood stream within minutes.

Timolol is a non-selective beta-adrenoreceptor antagonist. Oral timolol (20 - 30 mg daily) has been studied in three randomized controlled trials and have been found to reduce headache frequency by more than $50 \%$ when compared to placebo. ${ }^{3-5}$ It has been approved by FDA for prophylactic use in migraine patients and had Level A evidence to support this indication. The prophylactic benefit of betablockers in migraine treatment is not completely understood. It may be related to the effect of beta-blockers on central autonomic vascular tone center, which in turn modulate the cerebrovascular reactivity to sensory stimulation. ${ }^{11-12}$ Propranolol, a beta-adrenergic blocker modulates serotoninergic transmission, regulates periaqueductal pathway activation and prevents central sensitization, normalizes neuronal excitability in the CNS, and blocks cortical spreading depression. Topical ocular beta blockers when used daily have been reported to prevent migraine attacks. ${ }^{13-14}$

The specific aims in this study were to 1) assess the efficacy (headache freedom at 120 minutes) of timolol $0.5 \%$ ophthalmic solution compared to placebo in acute treatment of migraine headache; and 2) assess the safety and tolerability of timolol $0.5 \%$ ophthalmic solution in treatment of acute migraine headache.

The results of this randomized-controlled trial support that timolol $0.5 \%$ ophthalmic solution is not an efficacious treatment for acute migraine headache in this group of patients.

Our results are similar to a recent report by Cossack et al. ${ }^{15}$ They reported a randomized-controlled study of timolol in acute migraine. Ten patients participated that reported one to eight migraine attacks per month. In this series, the average percentage of headaches with a severity of none or mild at 120 minutes was $57 \%$ with placebo com-
$=0.26)$. In an exit survey, four patients found timolol highly effective compared to placebo and one patient reported placebo highly effective compared to timolol. The other five subjects did not report major differences between the two.

Therefore, while there is a good rationale for the use of topical ophthalmic beta-blocker medication for acute migraine, and prior case reports suggested such a benefit, we now have two randomizedcontrolled trials that did not demonstrate a marked effect of the drug compared to placebo. This is not to say that physicians may encounter individual patients who could report a beneficial effect of beta blocker eyedrops for acute migraine headaches. However, we could not demonstrate in a randomized-controlled trial the superiority of beta blocker eyedrops over placebo. Also, perhaps other formulations of beta blocker medications might be effective for treating acute migraine such as sublingual or intranasal. Finally, perhaps trials with a larger number of subjects with more power to detect a difference might show different results. Such a trial would require an external sponsor, either a pharmaceutical industry or the NIH.

\section{FUNDING SUPPORT}

This work was supported by a Ziegler Junior Investigator Grant from the Department of Neurology and a CTSA grant from NCATS awarded to the University of Kansas for Frontiers: University of Kansas Clinical and Translational Science Institute (\# ULlTR002366). The contents are solely the responsibility of the authors and do not necessarily represent the official views of the NIH or NCATS.

\section{REFERENCES}

1. Lipton RB, Bigal ME, Diamond M, Freitag F, Reed ML, Stewart WF. Migraine prevalence, disease burden, and the need for preventive therapy. Neurology 2007; 68(5):343-349. PMID: 17261680.

2. Baker JG. The selectivity of beta-adrenoceptor antagonists at the human betal, beta2 and beta3 adrenoceptors. Br J Pharmacol 2005; 144(3):317-322. PMID: 15655528.

3. Tfelt-Hansen P, Standnes B, Kangasneimi P, Hakkarainen H, Olesen J. Timolol vs propranolol vs placebo in common migraine prophylaxis: A double-blind multicenter trial. Acta Neurol Scand 1984; 69(1):1-8. PMID: 6367336.

4. Standnes B. The prophylactic effect of timolol versus propranolol and placebo in common migraine: Beta-blockers in migraine. Cephalalgia 1982; 2(3):165-170. PMID: 6758949 .

5. Stellar S, Ahrens SP, Meibohm AR, Reines SA. Migraine prevention with timolol. A double-blind crossover study. JAMA 1984; 252(18):2576-2580. PMID: 6387197.

6. Migliazzo CV, Hagan JC. Beta blocker eye drops for treatment of acute migraine. Mo Med 2014; 111(4):283-288. PMID: 25211851.

7. Hagan JC. Are drops the solution? A eureka moment? Beta blocker eye drops for acute migraines. Mo Med 2014; 111(4): 280-281. PMID: 25211850.

8. French BR, Singh NN. Beta blocking eyedrops in acute migraine: A novel use of an old drug. Mo Med 2014; 111(4):289-291. PMID: 25211852.

9. Dexter JK, Cady RK. Ophthalmic beta blockers: Treatment for acute migraine? Mo Med 2014; 111(4): 292-293. PMID: 25211853.

10. Chung SM. Can topical beta blockers be successful for acute migraine management? Mo Med 2014; 111(4):294-296. PMID: 25211854.

11. Min JH, Kwon HM, Nam H. The effect of propranolol on cerebrovascular reactivity to visual stimulation in migraine. J Neurol Sci 2011; 305(1-2):136-138. PMID: 21429523

12. Goadsby PJ, Charbit AR, Andreou AP, Akerman S, Holland PR. Neurobiology of migraine. Neuroscience 2009; 161(2):327-341. PMID: 19303917.

13. Bhagey J, James B. Topical timolol prevented migraine attacks. Eye (Lond) 2004; 18(7):751. PMID: 14765100.

14. Chiam PJ. Topical beta-blocker treatment for migraine. Int Ophthalmol 2012; 32(1):85-88. PMID: 22278763.

15. Cossack M, Nabrinsky E, Turner H, Abraham A, Gratton S. Timolol eyedrops in the treatment of acute migraine attacks: A randomized crossover study. JAMA Neurol 2018; 75(8):1024-1025. PMID: 29799915.

Keywords: migraine, headache, beta-blocker, opthamolic 